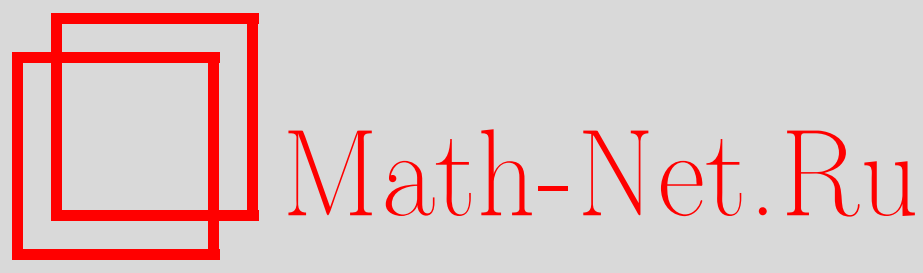

Ю. П. Чубурин, Квазиуровни двухчастичного оператора Шредингера с возмущенным периодическим потенциалом, ТМФ, 2009, том 158, номер 1, 115-125

DOI: https://doi.org/10.4213/tmf6302

Использование Общероссийского математического портала Math-Net.Ru подразумевает, что вы прочитали и согласны с пользовательским соглашением http://www . mathnet.ru/rus/agreement

Параметры загрузки:

IP : 35.173 .219 .149

26 апреля 2023 г., 13:08:09

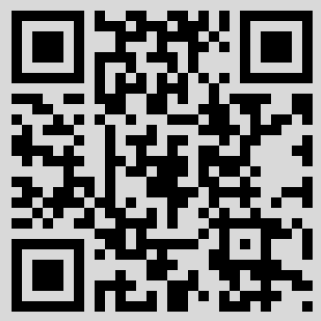




\title{
КВАЗИУРОВНИ ДВУХЧАСТИЧНОГО ОПЕРАТОРА ШРЕДИНГЕРА С ВОЗМУЩЕННЫМ ПЕРИОДИЧЕСКИМ ПОТЕНЦИАЛОМ
}

\begin{abstract}
Рассматривается двумерный периодический оператор Шредингера, возмущенный потенциалом взаимодействия двух (одномерных) частиц. Доказано существование квазиуровней (т.е. собственных значений или резонансов) данного оператора при фиксированном квазиимпульсе и малом возмущении вблизи граничных точек зон соответствующего периодического оператора. Исследовано асимптотическое поведение квазиуровней при стремлении к нулю константы связи. Получено простое условие того, что квазиуровень является собственным значением.
\end{abstract}

Ключевые слова: двухчастичный оператор Шредингера, периодический потенциал, собственное значение, резонанс.

\section{1. ВВЕДЕНИЕ}

В настоящей работе рассматривается оператор Шредингера вида

$$
H_{\varepsilon}=H_{0}+V(x)+\varepsilon W\left(x_{1}-x_{2}\right), \quad x=\left(x_{1}, x_{2}\right),
$$

действующий в пространстве $L^{2}\left(\mathbb{R}^{2}\right)$. Здесь $H_{0}=-\Delta, V(x)$ - вещественная ограниченная периодическая по обеим переменным функция с периодами, равными единице для простоты записи (в общем случае периоды должны быть соизмеримы), $\varepsilon>0$ (малый) параметр, $W\left(x_{1}\right)$ - вещественная функция, удовлетворяющая условию

$$
\left|W\left(x_{1}\right)\right| \leqslant e^{-a\left|x_{1}\right|}, \quad a>0 .
$$

В случае $V(x)=U\left(x_{1}\right)+U\left(x_{2}\right)$ гамильтониан $H_{\varepsilon}$ моделирует взаимодействие двух квантовых частиц в периодической среде.

Гамильтониан, подобный (1), рассматривался в работе [1], в которой для потенциалов нулевого радиуса действия и в рамках двухзонного приближения показано наличие связанного состояния при фиксированном квазиимпульсе и приведена “двухчастичная" интерпретация этого явления.

*Физико-технический институт УрО РАН, Ижевск, Россия.

E-mail: chuburin@otf.pti.udm.ru 
В настоящей статье в рамках строгого подхода доказано существование квазиуровней, т.е. собственных значений (резонансов), оператора $H_{\varepsilon}$ при фиксированном квазиимпульсе и малых $\varepsilon$. Исследовано асимптотическое поведение квазиуровней при $\varepsilon \rightarrow 0$. Получено простое условие того, что квазиуровень является собственным значением.

Заметим, что исследуемые в статье квазиуровни появляются в результате сдвига полюса функции Грина периодического оператора под действием возмущения (в результате межчастичного взаимодействия). В частности, если полюс лежит на границе зоны спектра периодического оператора (спектр такого оператора, как известно, состоит из зон - замкнутых промежутков), а квазиуровень является собственным значением, то в спектре исходного оператора в $L^{2}\left(\mathbb{R}^{2}\right)$ появляется соответствующая зона, отличная от зон, порождаемых периодическим потенциалом.

Также отметим, что наличие квазиуровней и характер их зависимости от квазиимпульса могут оказать существенное влияние на картину рассеяния в $\mathbb{R}^{2}$. Это связано с тем, что решения нестационарного уравнения Шредингера в $L^{2}\left(\mathbb{R}^{2}\right)$ (волновые пакеты), описывающие рассеивающиеся состояния, строятся как интегралы по квазиимпульсу от функции, имеющей в своем составе решение уравнения Липпмана-Швингера в ячейке, а решения уравнения Липпмана-Швингера имеют полюсы там, где находятся квазиуровни (ср. [2]).

В дальнейшем через $\sigma(A)$ будем обозначать спектр оператора $A$, через $\sigma_{\text {ess }}(A)-$ его существенный спектр.

\section{2. ФУНКЦИЯ ГРИНА ПЕРИОДИЧЕСКОГО ОПЕРАТОРА ШРЕДИНГЕРА}

В данном разделе приведены некоторые сведения об операторе $H=H_{0}+V(x)$, которые понадобятся в дальнейшем.

Введем обозначения $\Omega_{0}=[0,1]^{2}$ и $\Omega_{0}^{*}=[-\pi, \pi)^{2}$ для ячеек в прямой и двойственной решетках соответственно. Как известно [3], существуют семейства операторов $H_{0}(k)=-\Delta$ и $H(k)=H_{0}(k)+V(x)$, определенных в $L^{2}\left(\Omega_{0}\right)$ на (достаточно гладких) блоховских функциях и образующих разложения операторов $H_{0}$ и $H$ в прямом интеграле пространств

$$
\int_{\Omega_{0}^{*}}^{\oplus} L^{2}\left(\Omega_{0}\right) d k \cong L^{2}\left(\Omega_{0} \times \Omega^{*}\right),
$$

где $k \in \Omega_{0}^{*}-$ квазиимпульс. Под блоховскими функциями понимаются сужения на $\Omega_{0}$ функций $\psi(x)$, определенных на $\mathbb{R}^{2}$, таких, что

$$
\psi(x+n)=e^{i\langle k, n\rangle} \psi(x), \quad n \in \mathbb{Z}^{2}
$$

(через $\langle\cdot, \cdot\rangle$ обозначается скалярное произведение в $\mathbb{R}^{2}$ или $\mathbb{C}^{2}$ ). Оператор $H(k)$ имеет чисто дискретный спектр[3], его собственные значения, занумерованные с учетом кратности в порядке возрастания, будут обозначаться через $\lambda_{n}(k), n=1,2, \ldots$. 
В дальнейшем нам понадобится следующий явный вид унитарного оператора (преобразования Гельфанда):

$$
U: L^{2}\left(\mathbb{R}^{2}\right) \rightarrow L^{2}\left(\Omega_{0} \times \Omega_{0}^{*}\right), \quad \varphi(x) \mapsto \frac{1}{2 \pi} \sum_{n \in \mathbb{Z}^{2}} e^{i\langle k, n\rangle} \varphi(x-n),
$$

переводящего операторы $H_{0}$ и $H$ в соответствующие семейства

$$
U H_{0} U^{-1}=\left\{H_{0}(k)\right\}_{k \in \Omega_{0}^{*}}, \quad U H U^{-1}=\{H(k)\}_{k \in \Omega_{0}^{*}} .
$$

Введем следующие обозначения для резольвент:

$$
R(\lambda)=(H-\lambda)^{-1}, \quad R_{0}(k, \lambda)=\left(H_{0}(k)-\lambda\right)^{-1}, \quad R(k, \lambda)=(H(k)-\lambda)^{-1} .
$$

Функции Грина (я́дра резольвент) операторов $H_{0}(k)$ и $H(k)$ обозначим соответственно через $G_{0}(x-y, k, \lambda)$ и $G(x, y, k, \lambda)$. Имеем формулу

$$
G_{0}(x-y, k, \lambda)=\frac{1}{2 \pi} \sum_{n \in \mathbb{Z}^{2}} \frac{e^{i\langle k+2 \pi n, x-y\rangle}}{\langle k+2 \pi n, k+2 \pi n\rangle-\lambda} .
$$

Лемма 1. Предположим, что $\lambda_{0} \notin \sigma\left(H\left(k_{0}\right)\right)$. Тогда функиия Грина $G(x, y, k, \lambda)$ является аналитической $L^{2}\left(\Omega_{0} \times \Omega_{0}\right)$-значной функцией переменных $k, \lambda$ в некоторой комплексной окрестности точки $\left(k_{0}, \lambda_{0}\right) \in \mathbb{R}^{2} \times \mathbb{C} \subset \mathbb{C}^{2} \times \mathbb{C}$.

ДокАзАтЕЛЬство. Применяя равенство Парсеваля и (векторнозначную) теорему Вейерштрасса к ряду из аналитических функций (4), получаем, что $G_{0}(x-y, k, \lambda)$ является аналитической $L^{2}\left(\Omega_{0} \times \Omega_{0}\right)$-значной функцией при $\lambda \neq\langle k+2 \pi n, k+2 \pi n\rangle$, $n \in \mathbb{Z}^{2}$. Заменяя при необходимости $\lambda$ и $V(x)$ на $\lambda+\lambda^{\prime}$ и $V(x)+\lambda^{\prime}$ соответственно, можно считать, что $\lambda_{0} \neq\langle k+2 \pi n, k+2 \pi n\rangle$ для любого $n \in \mathbb{Z}^{2}$. В силу аналитической теоремы Фредгольма [4] операторнозначная функция $\left(1+R_{0}(k, \lambda) V\right)^{-1}$ аналитична всюду, кроме точек $\lambda \in \sigma(H(k))$. Теперь из равенства

$$
G(x, y, k, \lambda)=\left(1+R_{0}(k, \lambda) V\right)^{-1} G_{0}(x-y, k, \lambda)
$$

вытекает утверждение леммы.

Поскольку функция $G_{0}(x-y, k, \lambda)$ блоховская, из $(5)$ следует, что функция Грина $G(x, y, k, \lambda)$ естественным образом продолжается по переменным $x, y$ на $\mathbb{R}^{2} \times \mathbb{R}^{2}$, причем

$$
G(x+n, y, k, \lambda)=G(x, y-n, k, \lambda)=e^{i\langle k, n\rangle} G(x, y, k, \lambda), \quad n \in \mathbb{Z}^{2} .
$$

Далее, из равенств $U R(\lambda)=R(k, \lambda) U$ (см. (3)) и (6) вытекает соотношение

$$
G(x, y, k, \lambda)=\sum_{n \in \mathbb{Z}^{2}} e^{i\langle k, n\rangle} G(x-n, y, \lambda),
$$

где $\lambda \notin \sigma(H),(x, y) \in \mathbb{R}^{2} \times \mathbb{R}^{2}$. Из (7) получаем, что

$$
G(x, y, \lambda)=\frac{1}{(2 \pi)^{2}} \int_{\Omega_{0}^{*}} G(x, y, k, \lambda) d k .
$$




\section{3. ДВУХЧАСТИЧНОЕ ВЗАИМОДЕЙСТВИЕ}

Перейдем к новой ячейке $\Omega=\mathbb{R} \times[0,1]$. Введем унитарный оператор

$$
\begin{gathered}
U^{\prime}: L^{2}\left(\mathbb{R}^{2}\right) \rightarrow L^{2}\left(\Omega \times \Omega^{*}\right) \cong \int_{\Omega^{*}}^{\oplus} L^{2}(\Omega) d \kappa, \\
\varphi(x) \mapsto \frac{1}{\sqrt{2 \pi}} \sum_{\nu \in \mathbb{Z}} e^{i \kappa \nu} \varphi(x-(\nu, \nu))=\varphi^{\prime}(x, \kappa),
\end{gathered}
$$

где $\Omega^{*}=[-\pi, \pi), \kappa \in \Omega^{*}-$ квазимпульс. Очевидно,

$$
\varphi^{\prime}(x+(\mu, \mu), \kappa)=e^{i \mu \kappa} \varphi^{\prime}(x, \kappa), \quad \mu \in \mathbb{Z} .
$$

Легко видеть, что оператор $H_{\varepsilon}(1)$ расслаивается в прямом интеграле $(9)$ в семейство операторов

$$
H_{\varepsilon}^{\prime}(\kappa)=-\Delta+V(x)+\varepsilon W\left(x_{1}-x_{2}\right)
$$

формально такого же вида (1), но определенных на (достаточно гладких) функциях из $L^{2}(\Omega)$, являющихся сужениями функций, определенных на $\mathbb{R}^{2}$ и удовлетворяющих условию (10). Положим

$$
H^{\prime}(\kappa)=-\Delta+V(x), \quad R^{\prime}(\kappa, \lambda)=\left(H^{\prime}(\kappa)-\lambda\right)^{-1} .
$$

Обозначим через $G^{\prime}(x, y, \kappa, \lambda)$ функцию Грина оператора $H^{\prime}(\kappa)$. Предположим, что $\lambda \notin \sigma(H)$. Тогда имеет место равенство (ср. с разделом 2)

$$
G^{\prime}(x, y, \kappa, \lambda)=\sum_{\nu \in \mathbb{Z}} e^{i \kappa \nu} G(x-(\nu, \nu), y, \lambda)
$$

Лемма 2. Имеет место соотношение

$$
\sigma\left(H^{\prime}(\kappa)\right)=\bigcup_{k_{1}+k_{2}=\kappa} \sigma(H(k))
$$

ДокАЗАТЕЛЬство. Выберем в качестве ячейки в двойственной решетке для оператора $H(k)$ вместо $\Omega_{0}^{*}$ множество, ограниченное прямыми $k_{1}= \pm \pi, k_{2}=-k_{1} \pm \pi$. Запишем действие оператора $U$ (см. (3)) в виде

$$
\begin{aligned}
(U \varphi)(x, k) & =\frac{1}{2 \pi} \sum_{\left(n_{1}, n_{2}\right) \in \mathbb{Z}^{2}} e^{i\left(k_{1} n_{1}+k_{2} n_{2}\right)} \varphi\left(x-\left(n_{1}, n_{2}\right)\right)= \\
& =\frac{1}{2 \pi} \sum_{(\nu, \mu) \in \mathbb{Z}^{2}} e^{i \sigma \mu} e^{i \kappa \nu} \varphi(x-(\nu, \nu)-(\mu, 0)),
\end{aligned}
$$

где $\nu=n_{2}, \mu=n_{1}-n_{2}, \sigma=k_{1}, \kappa=k_{1}+k_{2}$. Из (13) следует, что оператор $U$ унитарно эквивалентен произведению двух унитарных операторов $U^{\prime \prime} U^{\prime}$, где оператор

$$
U^{\prime \prime}: L^{2}\left(\Omega \times \Omega^{*}\right) \rightarrow L^{2}\left(\Omega_{0} \times \Omega_{0}^{*}\right)
$$


задается формулой

$$
\left(U^{\prime \prime} \psi^{\prime}\right)(x, \kappa, \sigma)=\frac{1}{\sqrt{2 \pi}} \sum_{\mu \in \mathbb{Z}} e^{i \sigma \mu} \psi^{\prime}(x-(\mu, 0), \kappa) .
$$

Следовательно, операторы $H\left(k_{1}, \kappa-k_{1}\right), k_{1} \in[-\pi, \pi)$, образуют разложение оператора $H^{\prime}(\kappa)$ в прямом интеграле пространств

$$
\int_{[-\pi, \pi)}^{\oplus} L^{2}\left(\Omega_{0}\right) d k_{1}
$$

Из теории возмущений [3] следует, что собственные значения $\lambda_{n}\left(k_{1}, \kappa-k_{1}\right)$, $n=1,2, \ldots$, оператора $H\left(k_{1}, \kappa-k_{1}\right)$ непрерывно зависят от $k_{1}$. Отсюда и из теоремы XIII.85 из монографии [3] вытекает (12). Лемма доказана.

ЗАмЕчАниЕ 1. Обычным образом доказывается [3], что вдоль прямых $k_{1}+k_{2}=\kappa$ собственные значения оператора $H(k)$ не имеют участков постоянства и, следовательно, зоны спектра оператора $H^{\prime}(\kappa)$ невырожденны.

Лемма 3. Предположим, что $\lambda \notin \sigma\left(H^{\prime}(\kappa)\right)$. Тогда справедливо равенство

$$
G^{\prime}(x, y, \kappa, \lambda)=\frac{1}{2 \pi} \int_{-\pi}^{\pi} G\left(x, y,\left(k_{1}, \kappa-k_{1}\right), \lambda\right) d k_{1} .
$$

ДОКАЗАТЕЛЬСТВо. В силу (8), (11) и с учетом свойств блоховской функции $G(x, y, k, \lambda)$ имеем

$$
\begin{aligned}
G^{\prime}(x, y, \kappa, \lambda) & =\sum_{\nu \in \mathbb{Z}} e^{i \nu \kappa} \frac{1}{(2 \pi)^{2}} \int_{\Omega_{0}^{*}} G(x-(\nu, \nu), y, k, \lambda) d k= \\
& =\frac{1}{(2 \pi)^{2}} \int_{-\pi}^{\pi}\left(2 \pi \sum_{\nu \in \mathbb{Z}} e^{i \nu\left(\kappa-k_{1}\right)} \frac{1}{\sqrt{2 \pi}} \int_{-\pi}^{\pi} \frac{e^{-i \nu k_{2}}}{\sqrt{2 \pi}} G(x, y, k, \lambda) d k_{2}\right) d k_{1}= \\
& =\frac{1}{2 \pi} \int_{-\pi}^{\pi} G\left(x, y,\left(k_{1}, \kappa-k_{1}\right), \lambda\right) d k_{1} .
\end{aligned}
$$

Лемма доказана.

Лемма 4. Оператор умножения на функцию $W\left(x_{1}-x_{2}\right)$ представляет собой относительно компактное возмущение оператора $H^{\prime}(\kappa)$.

ДокАЗАТЕльство. В силу леммы 1 нормы в пространстве $L^{2}\left(\Omega_{0} \times \Omega_{0}\right)$ коэффициентов Фурье

$$
\frac{1}{\sqrt{2 \pi}} \int_{-\pi}^{\pi} e^{-i \nu k_{1}} G\left(x, y,\left(k_{1}, \kappa-k_{1}\right), i\right) d k_{1}
$$


экспоненциально убывают при $|\nu| \rightarrow \infty$. Учитывая (2), (14), запишем оценку

$$
\begin{aligned}
& \int_{\Omega} \int_{\Omega}\left|G^{\prime}(x, y, \kappa, i) W\left(x_{1}-x_{2}\right)\right|^{2} d x d y \leqslant \\
& \leqslant C \sum_{\mu \in \mathbb{Z}} \sum_{\nu \in \mathbb{Z}} \int_{\Omega_{0}} \int_{\Omega_{0}}\left|\frac{1}{2 \pi} \int_{-\pi}^{\pi} e^{i k_{1}(\mu-\nu)} G\left(x, y,\left(k_{1}, \kappa-k_{1}\right), i\right) d k_{1}\right|^{2} \times \\
& \quad \times e^{-a_{1}\left|x_{1}+\mu\right|} d x d y \leqslant C^{\prime} \sum_{\mu \in \mathbb{Z}}\left(\sum_{\nu \in \mathbb{Z}} e^{-a^{\prime}|\mu-\nu|}\right) e^{-a^{\prime \prime}|\mu|}<\infty
\end{aligned}
$$

где $a^{\prime}, a^{\prime \prime}>0$. Полученная оценка доказывает лемму.

Теорема 1. Справедливы следующие соотношения:

$$
\sigma_{\mathrm{ess}}\left(H_{\varepsilon}^{\prime}(\kappa)\right)=\sigma\left(H^{\prime}(\kappa)\right)=\bigcup_{k_{1}+k_{2}=\kappa} \sigma(H(k)) .
$$

Доказательство вытекает из лемм 2, 4 и из книги [3] (см. раздел XIII.4).

\section{4. КВАЗИУРОВНИ}

Предположим, что $\lambda_{0}=\lambda_{n_{0}}\left(k_{10}, \kappa-k_{10}\right)$ является невырожденным собственным значением оператора $H\left(k_{10}, \kappa-k_{10}\right)$, отвечающим нормированной собственной функции $\psi_{n_{0}}\left(x,\left(k_{10}, \kappa-k_{10}\right)\right)$. Можно считать, что $\lambda_{n_{0}}$ и $\psi_{n_{0}}$ аналитически зависят от параметра $k_{1}$ в комплексной окрестности $k_{10}$ (см., например, [3]), причем $\psi_{n_{0}}-$ как $L^{2}\left(\Omega_{0}\right)$-значная функция. В дальнейшем для простоты записи будут использоваться следующие обозначения:

$$
\widetilde{\psi}_{n_{0}}\left(x, k_{1}\right)=\psi_{n_{0}}\left(x,\left(k_{1}, \kappa-k_{1}\right)\right), \quad \tilde{\lambda}_{n_{0}}\left(k_{1}\right)=\lambda_{n_{0}}\left(k_{1}, \kappa-k_{1}\right),
$$

где $\kappa$ фиксировано.

Предположим далее, что

$$
\frac{\partial \widetilde{\lambda}_{n_{0}}\left(k_{10}\right)}{\partial k_{1}}=0, \quad \frac{\partial^{2} \widetilde{\lambda}_{n_{0}}\left(k_{10}\right)}{\partial k_{1}^{2}} \neq 0,
$$

а также, что конечно число точек $k_{1} \neq k_{10}$ таких, что $\tilde{\lambda}_{n}\left(k_{1}\right)=\lambda_{0}$ для некоторого $n$, и в них $\partial \widetilde{\lambda}_{n}\left(k_{1}\right) / \partial k_{1} \neq 0$. В частности, $\lambda_{0}$ может быть граничной точкой некоторой зоны спектра оператора $H^{\prime}(\kappa)$.

Положим $\xi=k_{1}-k_{10}$. Справедлива следующая лемма.

Лемма 5 [5]. Для $\lambda$ из некоторой комплексной окрестности точки $\lambda_{0}$ существуют ровно два корня $\xi_{j}=\xi_{j}(\lambda), j=1,2$, уравнения

$$
\widetilde{\lambda}_{n_{0}}\left(k_{10}+\xi\right)=\lambda
$$

совпадающие при $\lambda=\lambda_{0}$. Кроме того, существует аналитическая в окрестности нуля функция $\xi_{2}=\mu\left(\xi_{1}\right)$, причем $\mu^{\prime}(0)=-1$. 
В дальнейшем вместо спектрального параметра $\lambda$ будет часто использоваться параметр $\xi_{1}=\xi_{1}(\lambda)$ такой, что если $\left(\lambda-\lambda_{0}\right) \partial^{2} \widetilde{\lambda}_{n_{0}}\left(k_{10}\right) / \partial k_{1}^{2}>0$, то $\xi_{1}>0$. Соответственно будем пользоваться обозначениями вида $G^{\prime}\left(x, y, \kappa, \xi_{1}\right)$ вместо $G^{\prime}(x, y, \kappa, \lambda)$ и т.п.

Функции $\xi_{j}(\lambda), j=1,2$, порождают двулистное аналитическое накрытие окрестности точки $\lambda_{0}[6]$. При этом переходу параметра $\lambda$ на второй лист с верхней полуплоскости через вещественную ось отвечает переход одного корня в другой. Другими словами, после аналитического продолжения одного из корней, например $\xi_{1}$, в окрестность нуля происходит изменение знака $\operatorname{Im} \xi_{1}$ с плюса на минус. Это дает возможность рассматривать резонансы (см. ниже).

Пусть для определенности $\partial^{2} \widetilde{\lambda}_{n_{0}}\left(k_{10}\right) / \partial k_{1}^{2}>0$. В следующей лемме функция Грина $G^{\prime}(x, y, \kappa, \lambda)$ аналитически продолжена по параметру $\lambda$ в окрестности $\lambda_{0}$ с верхней полуплоскости через полуось $\left(\lambda_{0}, \infty\right)$ на второй лист (возможность такого продолжения видна из доказательства леммы). Соответственно предполагаем, что $\xi_{1}$ изменяется в окрестности нуля. Аналог следующей леммы для одночастичного "пленочного" оператора Шредингера см. в [5]. В дальнейшем предполагается, что $\sqrt{W}=\sqrt{|W|} \operatorname{sign} W$.

ЛЕмма 6. Для $\xi_{1}$ из достаточно малой комплексной окрестности нуля справедливо равенство

$$
G^{\prime}\left(x, y, \kappa, \xi_{1}\right)=\frac{i \widetilde{\psi}_{n_{0}}\left(x, k_{10}\right) \overline{\widetilde{\psi}_{n_{0}}\left(y, k_{10}\right)}}{\xi_{1} \partial^{2} \widetilde{\lambda}_{n_{0}}\left(k_{10}\right) / \partial k_{1}^{2}}+g\left(x, y, \kappa, \xi_{1}\right)
$$

При этом функиия

$$
\sqrt{\left|W\left(x_{1}-x_{2}\right)\right|} g\left(x, y, \kappa, \xi_{1}\right) \sqrt{W\left(y_{1}-y_{2}\right)}
$$

является $L^{2}(\Omega \times \Omega)$-значной аналитической функиией параметра $\xi_{1}$.

Доказательство аналогично доказательству леммы 2 в [5].

СледСтвиЕ. Операторнозначная функиия $\sqrt{|W|} R^{\prime}\left(\kappa, \xi_{1}\right) \sqrt{W}$ продолжается по параметру $\xi_{1}$ до мероморфной функиии аргумента $\xi_{1}$ в (комплексной) окрестности нуля. При этом (см. лемму 4) эта функиия принимает значения в множестве компактных операторов.

ЗАмЕчАниЕ 2. В силу мероморфной теоремы Фредгольма [3] и резольвентного тождества

$$
\left(1+\varepsilon \sqrt{|W|} R^{\prime}\left(\kappa, \xi_{1}\right) \sqrt{W}\right)^{-1}=1-\varepsilon \sqrt{|W|} R_{\varepsilon}^{\prime}\left(\kappa, \xi_{1}\right) \sqrt{W}
$$

операторнозначная функция $\sqrt{|W|} R_{\varepsilon}^{\prime}\left(\kappa, \xi_{1}\right) \sqrt{W}$ мероморфна по $\xi_{1}$ в окрестности нуля и принимает значения в множестве компактных операторов.

ЗАмечание 3 . В случае $V(x)=U\left(x_{1}\right)+U\left(x_{2}\right)$ имеем $\lambda_{n_{0}}\left(k_{1}, \kappa-k_{1}\right)=\lambda_{n_{1}}\left(k_{1}\right)+$ $\lambda_{n_{2}}\left(\kappa-k_{1}\right)$, где $\lambda_{n}\left(k_{j}\right)$ - собственные значения оператора $-d^{2} / d x_{1}^{2}+U\left(x_{1}\right)$ в ячейке $[0,1]$ для квазиимпульсов $k_{1}$ и $k_{2}=\kappa-k_{1}$. 
Положим $k_{1 j}=k_{10}+\xi_{j}, j=1,2$. Через $[x]$ обозначим целую часть числа $x \in \mathbb{R}$.

Аналог следующей леммы для одночастичного "пленочного" оператора Шредингера имеется в [7].

Лемма 7. Для всех достаточно малых (комплексных) $\xi_{1} \neq 0$ справедливо равенство

$$
\begin{aligned}
G^{\prime}\left(x, y, \kappa, \xi_{1}\right)= & \frac{i \widetilde{\psi}_{n_{0}}\left(x, k_{11}\right) \overline{\widetilde{\psi}_{n_{0}}\left(y, k_{11}\right)} \vartheta\left(\left[x_{1}\right]-\left[y_{1}\right]\right)}{\partial \widetilde{\lambda}_{n_{0}}\left(k_{11}\right) / \partial k_{1}}- \\
& \left.-\frac{i \widetilde{\psi}_{n_{0}}\left(x, k_{12}\right) \widetilde{\psi}_{n_{0}}\left(y, k_{12}\right)}{\partial \widetilde{\lambda}_{n_{0}}\left(k_{12}\right) / \partial k_{1}}+\left[y_{1}\right]-\left[x_{1}\right]\right) \\
&
\end{aligned}
$$

где $\vartheta(t)$ - функиия Хевисайда, а ү удовлетворяет оценке

$$
\left\|\gamma\left(x, y, \xi_{1}\right)\right\|_{L^{2}\left(\Omega_{0} \times \Omega_{0}+\left(\left[x_{1}\right],\left[y_{1}\right]\right)\right)} \leqslant C e^{-\sigma\left|\left[x_{1}\right]-\left[y_{1}\right]\right|}, \quad \sigma>0 .
$$

Назовем квазиуровнем оператора $H_{\varepsilon}^{\prime}(\kappa)$ полюс по переменной $\xi_{1}$ операторнозначной функции $\sqrt{|W|} R_{\varepsilon}^{\prime}\left(\kappa, \xi_{1}\right) \sqrt{W}$ в окрестности нуля (а также соответствующее значение спектрального параметра $\left.\lambda=\widetilde{\lambda}_{n_{0}}\left(k_{10}+\xi_{1}\right)\right)$. Назовем кратностъю квазиуровня $\xi_{1} \neq 0$ число $\operatorname{dim} \operatorname{ker}\left(1+\varepsilon \sqrt{|W|} R^{\prime}\left(\kappa, \xi_{1}\right) \sqrt{W}\right)$.

В силу (15) и аналитической теоремы Фредгольма [5] достаточно близкое к нулю число $\xi_{1} \neq 0$ является квазиуровнем тогда и только тогда, когда в классе $L^{2}(\Omega)$ существует ненулевое решение уравнения

$$
\varphi=-\varepsilon \sqrt{|W|} R^{\prime}\left(\kappa, \xi_{1}\right) \sqrt{W} \varphi .
$$

ТЕорема 2. Предположим, что

$$
\int_{\Omega} W\left(x_{1}-x_{2}\right)\left|\widetilde{\psi}_{n_{0}}\left(x, k_{10}\right)\right|^{2} d x \neq 0
$$

Тогда для всех достаточно мальх $\varepsilon>0$ существует ровно один квазиуровень $\lambda=$ $\widetilde{\lambda}_{n_{0}}\left(k_{11}\right)$ кратности единица, при этом

$$
\lambda=\lambda_{0}-\frac{\varepsilon^{2}\left(\int_{\Omega} W\left(x_{1}-x_{2}\right)\left|\widetilde{\psi}_{n_{0}}\left(x, k_{10}\right)\right|^{2} d x\right)^{2}}{2 \partial^{2} \widetilde{\lambda}_{n_{0}}\left(k_{10}\right) / \partial k_{1}^{2}}+O\left(\varepsilon^{3}\right) .
$$

Если, кроме того,

$$
\frac{\partial^{2} \widetilde{\lambda}_{n_{0}}\left(k_{10}\right)}{\partial k_{1}^{2}} \int_{\Omega} W\left(x_{1}-x_{2}\right)\left|\widetilde{\psi}_{n_{0}}\left(x, k_{10}\right)\right|^{2} d x<0,
$$

то квазиуровень является собственным значением.

ДокАзАтЕльство. Докажем первое утверждение теоремы. Пользуясь леммой 6 , перепишем уравнение (17) в виде

$$
\varphi(x)=-\frac{\varepsilon i \widetilde{\varphi}_{n_{0}}\left(x, k_{10}\right)}{\xi_{1} \partial^{2} \widetilde{\lambda}_{n_{0}}\left(k_{10}\right) / \partial k_{1}^{2}} \int_{\Omega} \overline{\widetilde{\varphi}_{n_{0}}^{\prime}\left(y, k_{10}\right)} \varphi(y) d y+\varepsilon A\left(\xi_{1}\right) \varphi(x),
$$


где $\widetilde{\varphi}_{n_{0}}=\sqrt{|W|} \widetilde{\psi}_{n_{0}}, \widetilde{\varphi}_{n_{0}}^{\prime}=\sqrt{W} \widetilde{\psi}_{n_{0}}, A\left(\xi_{1}\right)$ - это оператор с ядром $-\sqrt{|W|} g \sqrt{W}$. Положим для достаточно малых $\varepsilon$

$$
f=\left(1-\varepsilon A\left(\xi_{1}\right)\right) \varphi
$$

тогда в силу (19) $f=C \widetilde{\varphi}_{n_{0}}$, где $C=$ const. Отсюда вытекает, что в случае $\xi_{1} \neq 0$ существование ненулевого решения уравнения (17) эквивалентно существованию решения алгебраического уравнения

$$
\xi_{1}=-\frac{i \varepsilon\left(\left(1-\varepsilon A\left(\xi_{1}\right)\right)^{-1} \varphi_{n_{0}}, \widetilde{\varphi}_{n_{0}}^{\prime}\right)}{\partial^{2} \widetilde{\lambda}_{n_{0}}\left(k_{10}\right) / \partial k_{1}^{2}},
$$

где $(\cdot, \cdot)$ - скалярное произведение в $L^{2}(\Omega)$. В силу леммы 6 оператор $A\left(\xi_{1}\right)$ аналитически зависит от $\xi_{1}$ в окрестности нуля. Согласно теореме Руше уравнение (20) для всех достаточно малых $\varepsilon$ имеет единственное решение (квазиуровень) $\xi_{1}$. Разлагая $\left(1-\varepsilon A\left(\xi_{1}\right)\right)^{-1}$ в ряд по степеням $\varepsilon$ и пользуясь (20), получаем формулу

$$
\xi_{1}=\frac{\varepsilon}{i \partial^{2} \widetilde{\lambda}_{n_{0}}\left(k_{10}\right) / \partial k_{1}^{2}} \int_{\Omega} W\left(x_{1}-x_{2}\right)\left|\widetilde{\psi}_{n_{0}}\left(x, k_{10}\right)\right|^{2} d x+O\left(\varepsilon^{2}\right),
$$

при этом по условию теоремы $\xi_{1} \neq 0$. Из (21) вытекает (18). Утверждение о кратности следует из равенства $\varphi=C\left(1-\varepsilon A\left(\xi_{1}\right)\right)^{-1} \widetilde{\varphi}_{n_{0}}$.

Докажем второе утверждение теоремы. Пусть $\varphi \neq 0$ удовлетворяет уравнению (17), тогда функция

$$
\psi=-\varepsilon R^{\prime}\left(\kappa, \xi_{1}\right) \sqrt{W} \varphi=-\varepsilon R^{\prime}\left(\kappa, \xi_{1}\right) W \psi
$$

удовлетворяет уравнению Шредингера $H_{\varepsilon}^{\prime}(\kappa) \psi=\lambda \psi$, где $\lambda=\widetilde{\lambda}_{n_{0}}\left(k_{10}+\xi_{1}\right)$. Поэтому достаточно показать, что $\psi \in L^{2}(\Omega)$. В силу леммы 7

$$
\begin{aligned}
\psi(x)= & -\frac{i \varepsilon \widetilde{\psi}_{n_{0}}\left(x, k_{11}\right)}{\partial \widetilde{\lambda}_{n_{0}}\left(k_{11}\right) / \partial k_{1}} \int_{\Omega \cap\left\{\left[y_{1}\right] \leqslant\left[x_{1}\right]\right\}} \overline{\widetilde{\psi}_{n_{0}}\left(y, k_{11}\right)} \sqrt{W\left(y_{1}-y_{2}\right)} \varphi(y) d y+ \\
& +\frac{i \varepsilon \widetilde{\psi}_{n_{0}}\left(x, k_{12}\right)}{\partial \widetilde{\lambda}_{n_{0}}\left(k_{12}\right) / \partial k_{1}} \int_{\Omega \cap\left\{\left[y_{1}\right] \geqslant\left[x_{1}\right]\right\}} \overline{\widetilde{\psi}_{n_{0}}\left(y, k_{12}\right)} \sqrt{W\left(y_{1}-y_{2}\right)} \varphi(y) d y- \\
& -\varepsilon \int_{\Omega} \gamma\left(x, y, \xi_{1}\right) \sqrt{W\left(y_{1}-y_{2}\right)} \varphi(y) d y
\end{aligned}
$$

Пусть для определенности $x_{1} \geqslant 0$. Пользуясь (16), неравенством Коши-Буняковского и неравенством (2), оценим для $x \in \Omega_{0}$ и целого $n \geqslant 0$ интеграл

$$
\begin{aligned}
& \int_{\Omega_{0}}\left|\int_{\Omega} \gamma\left(x+(n, 0), y, \xi_{1}\right) \sqrt{W\left(y_{1}-y_{2}\right)} \varphi(y) d y\right|^{2} d x \leqslant \\
& \leqslant C \int_{\Omega_{0}}\left(\sum_{m \in \mathbb{Z}} \int_{\Omega_{0}}\left|\gamma\left(x+(n, 0), y+(m, 0), \xi_{1}\right)\right|^{2}\left|W\left(y_{1}-y_{2}+m\right)\right| d y\right) d x \leqslant \\
& \leqslant C_{1} \sum_{m \in \mathbb{Z}} e^{-2 \sigma|n-m|} e^{-a|m|}=C_{1}\left(e^{-2 \sigma n} \frac{1-e^{(2 \sigma-a)(n+1)}}{1-e^{2 \sigma-a}}+\right. \\
& \left.\quad+e^{2 \sigma n} \frac{e^{-(2 \sigma+a)(n+1)}}{1-e^{-(2 \sigma+a)}}+e^{-2 \sigma n} \frac{e^{-(2 \sigma+a)}}{1-e^{-(2 \sigma+a)}}\right)
\end{aligned}
$$


Данное выражение экспоненциально убывает при $n \rightarrow \infty$. Это же, очевидно, верно и при $n \rightarrow-\infty$.

Заметим, что в силу равенства

$$
\psi_{n_{0}}(x, k)=-\int_{\Omega_{0}} G_{0}\left(x-y, k, \lambda_{n_{0}}(k)\right) V(y) \psi_{n_{0}}(y, k) d y
$$

а также формулы (4) и неравенства Коши-Буняковского для комплексных $k$ из окрестности точки $k_{0} \in \Omega_{0}^{*}$ справедлива оценка

$$
\left|\psi_{n_{0}}(x, k)\right| \leqslant e^{\left|\operatorname{Im} k_{1}\right| \cdot\left|x_{1}\right|+\left|\operatorname{Im} k_{2}\right| \cdot\left|x_{2}\right|} .
$$

(Если $\lambda_{n_{0}}\left(k_{0}\right)=\left\langle k_{0}+2 \pi m, k_{0}+2 \pi m\right\rangle$ для некоторого $m$, то, как и выше, заменяем $\lambda$ и $V(x)$ на $\lambda+\lambda^{\prime}$ и $V(x)+\lambda^{\prime}$ для подходящего $\lambda^{\prime}$.)

Пусть $x_{1} \geqslant 0$ и $\left|\xi_{1}\right| \leqslant \delta<a / 2$. Запишем, пользуясь (25), оценку

$$
\begin{aligned}
& \left|\int_{\Omega \cap\left\{\left[y_{1}\right] \geqslant\left[x_{1}\right]\right\}} \overline{\widetilde{\psi}_{n_{0}}\left(y, k_{1 j}\right)} \sqrt{W\left(y_{1}-y_{2}\right)} \varphi(y) d y\right| \leqslant \\
& \leqslant C\left(\int_{\left[x_{1}\right]}^{\infty} e^{2 \delta y_{1}} e^{-a y_{1}} d y\right)^{1 / 2}=C \frac{e^{-(a-2 \delta)\left[x_{1}\right]}}{a-2 \delta}, \quad j=1,2 .
\end{aligned}
$$

Из формулы $(22)$, равенства $\vartheta\left(x_{1}-y_{1}\right)=1-\vartheta\left(y_{1}-x_{1}\right), x_{1} \neq y_{1}$, и оценок $(23),(26)$ вытекает, что для $x_{1} \geqslant 0$

$$
\psi(x)=-\frac{i \varepsilon \widetilde{\psi}_{n_{0}}\left(x, k_{11}\right)}{\partial \widetilde{\lambda}_{n_{0}}\left(k_{11}\right) / \partial k_{1}} \int_{\Omega} \overline{\widetilde{\psi}_{n_{0}}\left(y, k_{11}\right)} \sqrt{W\left(y_{1}-y_{2}\right)} \varphi(y) d y+\eta_{+}(x),
$$

где $\eta_{+} \in L^{2}\left(\Omega \cap\left\{x_{1} \geqslant 0\right\}\right)$. Аналогично для $x_{1} \leqslant 0$

$$
\psi(x)=\frac{i \varepsilon \widetilde{\psi}_{n_{0}}\left(x, k_{12}\right)}{\partial \widetilde{\lambda}_{n_{0}}\left(k_{12}\right) / \partial k_{1}} \int_{\Omega} \overline{\widetilde{\psi}_{n_{0}}\left(y, k_{12}\right)} \sqrt{W\left(y_{1}-y_{2}\right)} \varphi(y) d y+\eta_{-}(x),
$$

где $\eta_{-} \in L^{2}\left(\Omega \cap\left\{x_{1} \leqslant 0\right\}\right)$.

Используя (4), перепишем (24) для $k=\left(k_{1}, \kappa-k_{1}\right)$ в виде

$$
\begin{aligned}
& \psi_{n_{0}}(\left.x,\left(k_{1}, \kappa-k_{1}\right)\right)=-\frac{1}{2 \pi} e^{i\left\langle\left(k_{1}, \kappa-k_{1}\right), x\right\rangle} \times \\
& \quad \times \int_{\Omega_{0}}\left(\sum_{n \in \mathbb{Z}^{2}} \frac{e^{i\langle 2 \pi n, x-y\rangle} e^{-i\left\langle\left(k_{1}, \kappa-k_{1}\right), y\right\rangle}}{\left\langle\left(k_{1}, \kappa-k_{1}\right)+2 \pi n,\left(k_{1}, \kappa-k_{1}\right)+2 \pi n\right\rangle-\lambda_{n_{0}}\left(k_{1}, \kappa-k_{1}\right)}\right) \times \\
& \quad \times V(y) \psi_{n_{0}}\left(y,\left(k_{1}, \kappa-k_{1}\right)\right) d y .
\end{aligned}
$$

Отсюда следует, что $\left|\psi_{n_{0}}\right|$ экспоненциально убывает при $x_{1} \rightarrow \infty$ или $x_{1} \rightarrow-\infty$ в случае $\operatorname{Im} k_{1} x_{1}>0$ и экспоненциально возрастает в случае $\operatorname{Im} k_{1} x_{1}<0$. Второе утверждение теоремы следует теперь из соотношений $(21),(27),(28)$, а также леммы 5 и равенств $\operatorname{Im} k_{1 j}=\operatorname{Im} \xi_{j}, j=1,2$. Теорема доказана. 
ЗАмЕЧАниЕ 4 . В условиях теоремы 2 собственная функция $\psi(x)$ убывает в соответствии с неравенством

$$
\int_{\Omega_{0}}|\psi(x+(n, 0))|^{2} d x \leqslant C e^{-\gamma|n|}, \quad \gamma>0, \quad n \in \mathbb{Z} .
$$

ЗАМЕЧАНИЕ 5. Если

$$
\frac{\partial^{2} \widetilde{\lambda}_{n_{0}}\left(k_{10}\right)}{\partial k_{1}^{2}} \int_{\Omega} W\left(y_{1}-y_{2}\right)\left|\widetilde{\psi}_{n_{0}}\left(y, k_{10}\right)\right|^{2} d x>0
$$

и

$$
\int_{\Omega} \overline{\widetilde{\psi}_{n_{0}}\left(y, k_{1 j}\right)} \sqrt{W\left(y_{1}-y_{2}\right)} \varphi(y) d y \neq 0, \quad j=1,2,
$$

то, как и в доказательстве теоремы 2 , получаем, что функция $\psi(x)$ экспоненциально возрастает, если $\left|x_{1}\right| \rightarrow \infty$. Поскольку при этом $\operatorname{Im} \xi_{1}<0$, то $\xi_{1}$ является резонансом.

\section{Список литературы}

[1] S. M. Mahajan, A. Thyagaraja, J. Phys. A, 39:47 (2006), 667-671.

[2] Г. В. Вольф, Ю. П. Чубурин, ФТТ, 47:6 (2005), 1015-1018.

[3] М. Рид, Б. Саймон, Методы современной математической физики. Т. 4. Анализ операторов, Мир, М., 1982.

[4] М. Рид, Б. Саймон, Методы современной математической физики. Т. 1. Функционалъный анализ, Мир, М., 1977.

[5] Ю. П. Чубурин, ТМФ, 110:3 (1997), 443-453.

[6] Р. Ганнинг, Х. Росси, Аналитические функции многих комплексных переменных, Мир, M., 1969.

[7] Ю. П. Чубурин, ТМФ, 98:1 (1994), 38-47.

Поступила в редакцию 31.10 .2007 , после доработки 14.05.2008 